Research Article

\title{
Laboratorio de Innovación Social: escenario de participación, apropiación social e interdisciplinariedad
}

\section{Social Innovation Lab: scenario of social participation, social appropriation and interdisciplinarity}

\author{
Francisco Luis Giraldo-Gutiérrez, Luis Felipe Ortiz-Clavijo², Giovanni Alberto Zapata \\ Cardona $^{3}$ \\ ${ }^{1}$ Grupo de investigación CTS+i-, Instituto Tecnológico Metropolitano - ITM \\ ${ }^{2}$ Instituto Tecnológico Metropolitano - ITM \\ ${ }^{3}$ Colegio María Reina del Carmelo, Instituto Tecnológico Metropolitano - ITM \\ Correspondencia: ${ }^{1}$ franciscogiraldo@itm.edu.co, 2luisortiz250357@correo.itm.edu.co, \\ 3giovannizapata197311@correo.itm.edu.co
}

\begin{abstract}
Resumen: A partir de diferentes procesos llevados a cabo por el Instituto Tecnológico MetropolitanoITM con sede en la ciudad de Medellín (Colombia); durante el año 2019, se logra consolidar un espacio que ha permitido la cooperación entre el sector académico, el sector privado y la sociedad civil. Este artículo presenta una síntesis del camino recorrido, a partir de la exposición de los antecedentes y de algunos elementos conceptuales que dieron origen en el año 2012 al Laboratorio de Innovación Social - LIS. Posteriormente, se presentan diversas metodologías que pueden ser aplicadas en laboratorios de esta categoría y se da a conocer el modelo de trabajo del LIS en donde se establecen tres ejes, a saber: la apropiación social del conocimiento; la apropiación social de la ciencia, la tecnología y la innovación, y la innovación social como eje dinamizador en el marco de la cuarta revolución industrial.
\end{abstract}

Palabras clave: laboratorio de innovación social, apropiación social del conocimiento, innovación social, desarrollo socioeconómico.

\begin{abstract}
Different processes carried out in 2019 by the Metropolitan Technological Institute - ITM, located in the city of Medellin (Colombia), have enabled to consolidate a space for cooperation between academic sector, private sector, and civil society. This article presents a synthesis of the path followed through the explanation of the background and some conceptual elements that had given rise to the Social Innovation Lab - LIS in 2012. Subsequently, several methodologies are presented in order to show different applications in the context of this kind of labs. Finally, the paper presents the LIS working model, in which three axes are established: the social appropriation of knowledge; the social appropriation of science, technology, and innovation; the social innovation as a catalyst in the framework of the fourth industrial revolution.
\end{abstract}

Keywords: social innovation lab, social appropriation of knowledge, social innovation, socioeconomic development. 


\section{Introducción}

La discusión teórica sobre fenomenología de la innovación ha sido un tema recurrente, el acervo documental e investigativo disponible deja en evidencia que la discusión se concentró inicialmente en cuatro clases de innovación, a saber: innovación de aplicaciones, innovación de producto, innovación de proceso y la innovación de modelo de negocio. De esta primera concentración, se despliegan subtipos que buscan dar respuesta en términos de clasificación al exponencial crecimiento en los diferentes ámbitos de producción de la sociedad. De ahí que, se consolidaran esfuerzos para medir las actividades científicas, tecnológicas y de desarrollo, de la mano de la Organización para la Cooperación y el Desarrollo Económico (OCDE), la cual promueve una serie de directrices para los Estados miembros, compiladas en manuales como Frascati (1963) y Oslo (1997).

Por su parte, otros organismos e iniciativas internacionales tales como: la Organización de los Estados Americanos (OEA), la Red de Indicadores de Ciencia y Tecnología -Iberoamericana e Interamericana (RICYT) y el Programa Iberoamericano de Ciencia y Tecnología para el Desarrollo (CYTED) han promovido un manual homólogo a los mencionados, denominado Manual de Bogotá, enfocado a la normalización de Indicadores de Innovación Tecnológica en América Latina y el Caribe.

Llegados a este punto, observamos como la abundante documentación guía establece una normalización que puede aplicarse a las innovaciones. Sin embargo, algunos términos relacionados con la innovación han tomado relevancia en los últimos años, como es el caso de la innovación social, un término usado para referirse a un amplio espectro de soluciones innovadoras a problemas sociales, de ahí que la discusión alrededor de una taxonomía sea de gran relevancia, en concordancia con (Monge \& Allamand, 2016) al señalar la existencia de una creciente discusión acerca de la definición y alcance de innovación social, dando lugar a diferentes interpretaciones y enfoques metodológicos.

Ahora bien, para dar cumplimiento a estas prerrogativas administrativas y corrientes teóricas, en el marco del Plan de Desarrollo 2012-2015 del Instituto Tecnológico Metropolitano ITM- de la ciudad de Medellín, se creó un Sistema Integrado de Laboratorios Científicos Parque I. que contó en su momento con 23 laboratorios, hoy ya suma 25. Dentro de este Sistema se crea el Laboratorio de Innovación Social, en adelante LIS. En este sentido, en el presente artículo se evidencia cuál y cómo ha sido la consolidación del LIS, como un proyecto de ciudad con proyección nacional e internacional.

De este modo, se parte por presentar una breve conceptualización de la innovación y particularmente de la innovación social para tener claro cuál es el concepto que se concibe desde el LIS. A continuación, se presenta el LIS como una experiencia académica e investigativa, dejando ver su nacimiento, lo qué hace, cómo lo hace y algunas experiencias de experimentación o participación en proyectos. Finalmente, se esbozan algunas áreas de trabajo que presentan al LIS como un centro de investigación y un aliado estratégico para la contribución social en el marco de los Objetivos de Desarrollo Sostenible - ODS.

\section{Innovación, transformación y desarrollo socioeconómico}

El conocimiento ha sido, a lo largo del proceso evolutivo y constitutivo de las sociedades, el capital de desarrollo y transformación socioeconómica. El conocimiento, ha sido la materia prima para el desarrollo científico y tecnológico, en todos los períodos de la humanidad y en especial, después de la primera Revolución Industrial. Es así como, desde el siglo XVIII, los pequeños poblados y aldeas, no han parado de crecer y urbanizarse hasta convertirse hoy en grandes urbes con gran potencial de generación de conocimiento y de desarrollo industrial, comercial o de servicios.

En dicho proceso de industrialización, como eje vital del desarrollo económico por su aporte al progreso técnico y a la elevación de la productividad, la combinación de aprendizaje e innovación adquiere mayor importancia. De esta manera, hay que reconocer que una de las 
características del proceso de industrialización de América Latina hasta ahora ha sido precisamente la asimetría entre un elevado componente de imitación (fase previa del aprendizaje) y un componente marginal de innovación económico-social (Fajnzylber, 1990, p. 8).

Es en esta línea, que, desde finales del 60 y durante toda la década del 70 del siglo XX, se establecieron los planes y programas de ciencia y tecnología para América Latina y el Caribe, con el propósito de ser competitivos con los países desarrollados y comenzar a pensar y producir nuestro propio conocimiento científico y tecnológico. No obstante, los planes y programas, estas plataformas, estos sistemas de desarrollo científico y tecnológico, requieren de inversiones y recursos permanentes y ascendentes, para alcanzar los niveles de desarrollo que tienen los países industrializados. Los sistemas de producción y desarrollo científico, tecnológico e industrial, seguirán siendo obsoletos, si no hay una inversión directa del Estado y el sector productivo. Reiterando, vemos que:

"Desde fines del decenio de 1970 en los países industrializados se ha venido validando la concepción de que el cambio tecnológico desempeña una función esencial en las políticas de cambio estructural. En la reunión de la cumbre celebrada en Tokio, en mayo de 1986, se recalcó la necesidad de aplicar políticas eficaces de reajuste estructural en todos los países y en todas las actividades económicas, con el objeto de promover el crecimiento económico, el empleo y la integración de las economías internas en la economía mundial. Esas políticas deben comprender la innovación tecnológica, la adaptación de la estructura industrial y la expansión del comercio y de la inversión extranjera directa" (Fajnzylber, 1990, p. 49).

Si bien el desarrollo tecnológico, y la producción de bienes y servicios de base tecnológica, desde la primera Revolución Industrial ha transformado las formas de vivir, de relacionarse de las personas consigo mismas y con su entorno, también es evidente la transformación y el fortalecimiento de un nuevo modelo económico y de producción industrial, de acumulación de riqueza, que aumenta la población en condiciones de pobreza y pobreza extrema. Los niveles de inequidad, de desabastecimiento de agua potable y alcantarillado para un número no despreciable de los ciudadanos, sigue siendo una constante. Pese a que el desarrollo tecnológico se acogió como la salida de las condiciones de miseria, hoy la situación es otra, el desarrollo tecno-científico ha aumentado las brechas de desigualdad e inequidad social, económica y política.

De ahí que la innovación social emerja como un enfoque válido asociado a características endógenas y a una serie de aspectos estructurales y variables sociales que le son propias a cada contexto. Las actuales tendencias para la generación de capacidades de innovación, parten de identificar aquellos factores que logren integrar funciones productivas con la generación de conocimientos, de la existencia de organismos capaces de asumir los riesgos que la innovación supone, de un nuevo rol del Estado mucho más activo y del diseño de instrumentos para la transferencia de tecnología. En definitiva, se requiere un cambio cultural frente a una nueva forma de competir en los mercados. La innovación es vista como un fenómeno social, en el cual la capacidad de cooperar o complementar funciones se vuelve un factor determinante del éxito.

En los actuales debates de innovación, transformación y desarrollo socioeconómico, se sostiene que desarrollar procesos innovadores supone definir nuevos roles, producir cambios culturales y nuevas actitudes, redefinir el papel del Estado frente a la ciencia y la tecnología, conformar verdaderas redes de innovación social, complementariedad productiva y desarrollar nuevos instrumentos destinados a atender las necesidades de los grupos sociales en el territorio. Todo esto exige claridad en el proceso y las metodologías a emplear. Es necesario diseñar e implementar planes que acompañen los cambios que suelen ser de largo plazo. Para ello los gobiernos deben asumir un papel mucho más activo en materia de apoyo a la competitividad, un papel que supone crear las condiciones favorables para la innovación, atendiendo y profesionalizando los instrumentos para considerar realidades muy disímiles, incluso dentro de un mismo país, atendiendo diferencias desde un punto de vista sectorial o territorial. Se 
requiere profesionalizar, rediseñar, redefinir y reorientar los instrumentos puestos a disposición de las empresas y de aquellos pensados para intermediar entre la generación de nuevos conocimientos (universidades, laboratorios, institutos tecnológicos, centros de investigación y desarrollo, parques tecnológicos, etc.) y las empresas.

\section{El Laboratorio de Innovación Social: escenario de participación, apropiación social e interdisciplinariedad}

Como se ha planteado, el LIS, nace en el marco del Plan de Desarrollo Institucional 20122015 del Instituto Tecnológico Metropolitano - ITM, situado en la ciudad de Medellín (Colombia), siendo una institución de educación superior con orientación, vocación y tradición tecnológica, de carácter público y de orden municipal.

En el marco del Plan de Desarrollo Institucional 2012-2015 se incluyó un componente denominado "Parque I", el cual comprende en buena medida una infraestructura que tiene el músculo no solo tecnológico sino y sobre todo social, desde la investigación científica para asumir los retos de ciudad, región y país y dar respuesta a los distintos fenómenos, sociales, políticos, económicos, ambientales, culturales del territorio que se presentan en los distintos escenarios.

Esto nos lleva a plantear que desde la concepción del ITM, como un centro de formación y su posterior proyección como Institución de Educación Superior, el inmediato antecedente del LIS fue el Centro de Estudios Ciudad de Medellín (CECIM), que operó como centro de reflexión sobre temas urbanos y también como grupo de investigación desde 2004. En el periodo rectoral 2012-2015, se decidió darle un giro al Centro buscando que sus actividades tuvieran una incidencia social más directa y permanente en el mejoramiento de la calidad de vida de la ciudad, con vocación tecnológica e innovadora, con lo cual se decidió a comienzos del 2012 crear el LIS, concebido como un laboratorio donde se maduran y desarrollan proyectos de innovación social, especialmente en formación para el empleo productivo y la creación de empresas sociales, el fortalecimiento de la cultura urbana y el fomento de la participación ciudadana.

El Laboratorio de Innovación Social, es un espacio académico diseñado dentro de los laboratorios de investigación del ITM para la idealización, desarrollo e implementación, de proyectos, planes, programas y acciones de corte socio-humanístico o que guarden relación y tengan este componente. En este sentido, se proyecta como un espacio transversal y de aliado estratégico, para las acciones que se desarrollen en todas las áreas del conocimiento científico, tecnológico y de innovación, de gestión de lo público y lo privado.

Como instancia investigativa y académica, el LIS, tiene como misión estar comprometido con el desarrollo de Medellín, Antioquia y el País, mediante la producción, adaptación, difusión y uso de conocimiento científico y tecnológico. Con sus aportes propende por la transformación productiva y cultural de los territorios, socialmente incluyente y respetuosa de la vida y el medio ambiente. Así mismo, se proyecta con una Visión para el 2021 de ser reconocido nacional e internacionalmente como un laboratorio innovador por sus aportes al mejoramiento de la calidad de vida y el desarrollo humano de los habitantes de la ciudad, la región y el país.

Como se aprecia, tanto la misión como la visión son coherentes, consecuentes y pertinentes, no solo con una realidad local, sino que se articula con las realidades, situaciones y condiciones del orden regional y nacional, y se sustenta con las tendencias mundiales, especialmente con aquellas planteadas desde entidades como el Banco Interamericano de Desarrollo - BID, la UNESCO y la ONU.

Para dar cumplimiento la misión y la visión, el LIS tiene como acciones puntuales las siguientes:

- Formulación de proyectos de investigación en diversas convocatorias de orden nacional e internacional.

- Formulación de proyectos basados en las necesidades de los usuarios. 
- Investigación formativa.

- Conformación de redes y alianzas estratégicas.

- Generación de espacios académicos e investigativos en temas relacionados con la paz y el posconflicto.

- Documentación y teorización de líneas de investigación asociadas a la Innovación Social.

Los aspectos anteriores son posibles dado lo siguiente:

- Sistematización, modelamiento y simulación de experiencias, planes, programas que se desarrollan, evidenciando el impacto social, ambiental, económico y político de los mismos.

- Diseño de proyectos de investigación aplicada.

- Diseño de proyectos de innovación social, especialmente aquellos que pretenden un amplio desarrollo y transformación social.

- Co - creación de soluciones sociales alternativas.

- Elaboración de diagnósticos en contextos educativos, sociales, políticos, culturales, recreativos, ambientales.

- Diseño y prototipado.

- Difusión y escalamiento de soluciones sociales.

- Asesorías y consultorías haciendo uso de metodologías propias de innovación abierta, orientada al desarrollo sostenible.

Hay que señalar que uno de los grandes desafíos que ha tenido el equipo del LIS tiene que ver con la definición de su foco de actuación. Para el año 2013, pretendía proyectarse como un centro de proyección social del ITM a la ciudad-región metropolitana de Medellín, mediante el uso de la tecnología y la formación tecnológica en la solución de problemas que entran en sus áreas de actuación: emprendimiento social, formación empresarial, y fortalecimiento de identidades urbanas por medio del arte y la cultura. Para lograrlo contempla varias estrategias:

- Ampliación de su capital humano mediante alianzas estratégicas con grupos de investigación y centros de desarrollo tecnológico, internos y externos.

- Alineación permanente con el proyecto Sinergia (proyecto de la Alcaldía de la ciudad de Medellín, en el cual se integran las tres instituciones de educación superior de carácter público adscritas a la municipalidad, siendo estas: la Institución Universitaria Colegio Mayor de Antioquia, la Institución Universitaria Pascual Bravo y el Instituto Tecnológico Metropolitano -ITM).

- Alineación con los objetivos de los programas como la U en Mi Barrio y la U en Mi Empresa.

- Participación en redes de innovadores sociales de diversa procedencia geográfica y cultural.

- Participación en convocatorias públicas y privadas derivadas de programas sociales contemplados en los planes de desarrollo municipal y departamental.

- Ampliación y diversificación de fuentes de financiamiento.

- Visibilidad a través de la participación en eventos académicos, publicaciones y un uso intensivo de las redes sociales electrónicas.

- Transferencia y escalamiento de resultados de proyectos sociales innovadores.

Cabe señalar que, un hito importante, el primero, que comenzó a marcar el devenir del LIS, fue la identificación de cuatro áreas de trabajo estratégicas para el ITM, la ciudad, la región y el país, asumidas desde el laboratorio. Estas son: economía, emprendimientos sociales y empleo productivo; educación, derechos humanos e inclusión social; arte, cultura e identidad de comunidades urbanas y rurales; y territorio, urbanismo y sostenibilidad ambiental. Áreas que no solo integran los distintos tópicos problema, transversales a toda ciencia y/o disciplina, sino que posibilitan la investigación e intervención de manera sistémica, holística y heurística. 
En esa misma línea de pensamiento y accionar del LIS se constituye como un espacio que posibilita la relación de la Ciencia, la Tecnología y la Sociedad, desmarcándose del imaginario de laboratorio provisto con equipos, de esta forma:

"No es un lugar físico provisto de equipos, sistemas de medición y personal científico y de apoyo, sino un laboratorio de laboratorios, esto es, un espacio abierto de comunicación y colaboración donde los recursos y las capacidades científicas y tecnológicas del ITM se ponen al servicio del mejoramiento de la calidad de vida de los habitantes de Medellín y su Área Metropolitana, mediante el uso de metodologías participativas de diagnóstico y diseño" (ITM, 2019).

Para lograr este propósito, se ha diseñado una metodología para el diseño y ejecución de proyectos, cuyo rasgo diferencial es la innovación abierta a la co-creación. Existe un número amplio de prácticas sociales, académicas y metodológicas, concebidas como estrategias para generar capacidades de innovación social. En la Tabla 1, se relacionan algunas de las metodologías aplicables a un laboratorio de Innovación Social, las cuales fueron punto de partida para la determinación del modelo metodológico del LIS.

Tabla 1. Metodologías aplicables a laboratorios de Innovación social

\begin{tabular}{|c|c|}
\hline Nombre & Descripción \\
\hline Metodología Canvas & $\begin{array}{l}\text { Esta metodología de innovación y diseño incluye un lienzo (Canvas) con } 9 \\
\text { elementos que parten de determinar la oferta de valor frente a la segmentación } \\
\text { de clientes de la empresa u organización. De ahí se clarifican los canales de } \\
\text { distribución y las relaciones, todos estos determinan los beneficios e ingresos. } \\
\text { Después, se especifican los recursos y las actividades esenciales que } \\
\text { determinan los costos más importantes (De la Mata, 2011). }\end{array}$ \\
\hline Design Thinking & $\begin{array}{l}\text { Es una metodología que permite generar ideas innovadoras que centra su } \\
\text { eficacia en entender y dar solución a las necesidades reales de los usuarios. } \\
\text { Proviene de la forma en la que trabajan los diseñadores de producto. De ahí su } \\
\text { nombre, que en español se traduce de forma literal como "Pensamiento de } \\
\text { Diseño" (Muñoz, 2017, p. 23). }\end{array}$ \\
\hline $\begin{array}{l}\text { Innovation Lab (I-Lab) } \\
\text { del Banco Interamericano } \\
\text { de Desarrollo (BID) }\end{array}$ & $\begin{array}{l}\text { Metodología de la innovación social promovida por el Banco Interamericano } \\
\text { de Desarrollo BID (2017) generada desde plataformas donde los propios } \\
\text { ciudadanos definen y priorizan sus problemas, y se conectan con quienes } \\
\text { tienen las capacidades, intereses y recursos para atenderlos: gobiernos, } \\
\text { empresas, universidades y ONG a nivel local, regional y mundial. Fases: } \\
\text { - Concurso de problemas } \\
\text { - Priorización de necesidades } \\
\text { - Concurso de soluciones } \\
\text { - Selección de la mejor solución } \\
\text { - Implementación de las soluciones }\end{array}$ \\
\hline $\begin{array}{ll}\text { Proceso } & \text { metodológico } \\
\text { prospectivo } & \end{array}$ & $\begin{array}{l}\text { El Plan estratégico con enfoque prospectivo promovido por el Colegio Mayor } \\
\text { de Antioquia (2018), se caracteriza por los enfoques sistémicos, holísticos y de } \\
\text { largo plazo con los que realiza el proceso formulación, porque busca articular } \\
\text { su sostenibilidad y desarrollo con las demás dinámicas municipales, } \\
\text { regionales, nacionales e internacionales; buscando dar respuesta a la real } \\
\text { problemática a solucionar y a enfrentar los retos y cambios; con el fin de } \\
\text { visualizar un modelo de sostenibilidad y permanencia de futuro, mediante un } \\
\text { ejercicio de reflexión y construcción colectiva. Fases: Fase 1: Estado } \\
\text { Situacional, Fase 2: Formulación estratégica, Fase 3: Diseño Estratégico. }\end{array}$ \\
\hline $\begin{array}{l}\text { Metodología Solución } \\
\text { Creativa de Problemas } \\
\text { Sociales (SCPS) }\end{array}$ & $\begin{array}{l}\text { Esta nueva metodología fortalece el proceso de innovación social desde el } \\
\text { potencial creativo que todas las personas tienen, el protagonismo, la } \\
\text { participación y la inclusión de todo tipo de agentes sociales vinculados a un } \\
\text { mismo Reto Social, generando experiencias enriquecedoras, prácticas y } \\
\text { enfoques innovadores para mejorar la calidad de vida de las personas. De este } \\
\text { mismo proceso participativo y creativo surge el Juego Twist thinking, un }\end{array}$ \\
\hline
\end{tabular}


juego de cartas dirigido a despertar el espíritu emprendedor, la creatividad y la motivación de los más jóvenes decididos por el cambio en el entorno social, aprendiendo metodologías, herramientas y dando forma a las ideas con un valor añadido, la innovación social (Jovesólides, 2020).

Laboratorios ciudadanos $\quad$ En los últimos años han surgido una gran cantidad de nuevas maneras de organizar encuentros, actividades, debates y talleres en los que no hay un programa establecido, sino que son los propios participantes quienes deciden activamente las actividades que los configurarán, en algunos casos con antelación mediante la ayuda de plataformas online como wikis y listas de correo, y en otros decidiéndolo in situ en el momento del encuentro. A estas nuevas metodologías se las conoce como tecnologías de los espacios abiertos y se aplican en la organización de eventos de distinta naturaleza como las unconferences, BarCamps o hack meetings. Algunas metodologías se configuran como talleres que están orientados a la producción de proyectos que se llevan a cabo por los propios participantes en el encuentro: startup weekends, game jams, open labs, hackatones, etc. (Serra, 2010).

La metodología DIA Debe estar aplicada en la realización de los proyectos innovadores del (UNAL) Laboratorio de Innovación para la Paz, propuesta por la Universidad Nacional de Colombia (2018). Esta se divide en 4 etapas:

Dream Hub: Fundamentos. Se concibe una idea innovadora y se recolecta información para dar alcance a esa idea o problemática (lluvia de ideas). En esta etapa un experto evalúa cuan factibles es la realización de la idea.

Shape Hub: Proceso creativo. Se plasma la idea y se dan posibles soluciones para desarrollar el proyecto. Se definen los procesos y actividades a realizar para implementar el proyecto. En esta etapa se debe dar inicio la capacitación en temas de paz, innovación y tecnología a los jóvenes participantes. El resultado de la etapa es un plan de acción del proyecto innovador.

Make Hub: En esta etapa se debe realizar el proyecto, es decir, se realizan las pruebas necesarias hasta producir un prototipo viable. Finalmente se hace la presentación del proyecto.

Link Hub: Se debe compartir el producto final con la comunidad y con inversionistas interesados para hacer crecer y desarrollar la idea. Se debe asesorar al participante en emprendimiento.

Fuente: Elaboración propia, 2020

\section{Ejes de trabajo del Laboratorio de Innovación Social LIS del ITM}

En el LIS se identifican varios frentes y áreas de trabajo específicos, no obstante, se concibe y practica el concepto de innovación social, en sentido amplio, esto es, no se reduce a la elaboración de productos o servicios con enfoque social, sino, además, a aquellas prácticas sociales y acciones de grupo, organizados o no, que contribuyen al desarrollo y la transformación social de manera efectiva.

Entre los ejes de trabajo se encuentran aquellos que se corresponden con la apropiación Social, acogiendo en principio los referentes de la Estrategia Nacional de Apropiación Social, planteada por Colciencias (hoy Minciencias) hacia el año 2005.

En este sentido, la Apropiación Social, ha sido ampliamente discutida por diversos autores quienes han tratado de llegar a un consenso en su definición, sin embargo, el alcance del concepto no se limita a una noción única. Con este propósito, la gráfica 1 presenta las dimensiones de apropiación que se consideran en esta investigación. 
Figura 1. Dimensiones de la apropiación

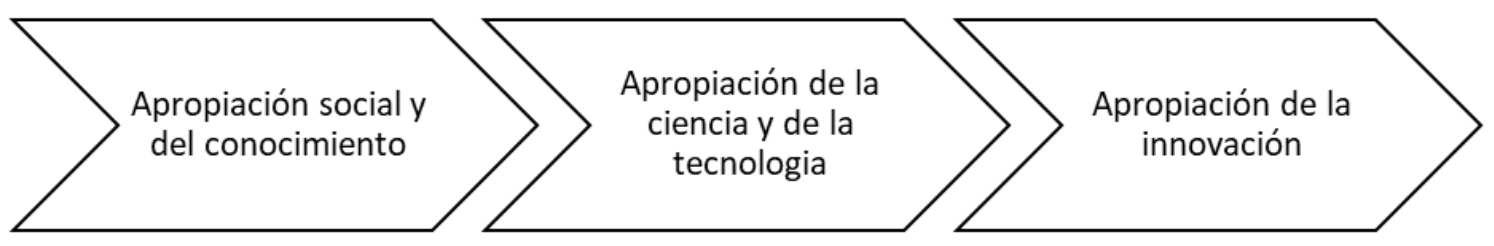

Fuente: Giraldo Gutiérrez et al., 2020, p. 312.

Es así como, el concepto de apropiación social y apropiación social del conocimiento, ha sido uno de los escenarios de mayor discusión en relación con su origen o su definición, en primer lugar:

"El origen del concepto de "apropiación social" se remonta a las ciencias sociales y se configura como una consecuencia de popularizar los conocimientos, de divulgar resultados de investigaciones en los distintos ámbitos del saber y analizar los diferentes casos de estudio" (Giraldo Gutiérrez et al., 2020, p. 312).

La apropiación Social se ubica y acepta, como el medio de divulgación y comunicación de las acciones, hechos y desarrollos de la ciencia y la tecnología, no obstante, es claro que antes de poder tener evidencia de algo para contar, para divulgar o informar, debe producirse el hecho mismo. Como hecho, la apropiación social deja ver el nivel de aplicabilidad, de usos, de consumo y de transformación que hace una comunidad o grupo social de los desarrollos científicos y tecnológicos, y de cómo, con esas transformaciones de CTI se generan cambios, desarrollos y transformaciones, se generan bienes y servicios que favorecen a un gran número de habitantes de una ciudad o comunidad.

Otro eje de trabajo, que en cierta medida deviene, no de manera exclusiva, pero si más validada, valorada y en ocasiones reconocida, es la generación de conocimiento. El conocimiento en sí, es generado por comunidades naturales, grupos de personas que se reúnen espontáneamente en torno a una idea, necesidad, vivencia, problema y generan un conocimiento de ese problema o necesidad, que los lleva a la solución. Dicho conocimiento, también es generado de manera sistemática en la Universidades, con sus programas formativos, sus semilleros de investigación, los grupos y líneas de investigación. De igual manera, se generan conocimientos en los centros de Investigación y Desarrollo, en los Departamentos de I+D de empresas. En el LIS entendemos que la transferencia del conocimiento es una de las funciones esenciales de la academia y en concordancia con esto, reconocemos como concepto de transferencia del conocimiento el propuesto por Bayona y González (2010) quienes la definen como un proceso de interacción social orientado hacia la producción y circulación de conocimiento que genera externalidades de aprendizaje. Este proceso interactivo es interno y externo a la organización, combina distintas capacidades y recursos organizacionales. Dicha transferencia no puede ser analizada fuera del contexto social específico en el que tiene lugar. Las condiciones del entorno moldean, facilitan u obstaculizan las relaciones de transferencia de conocimiento. Asimismo, Murray et al. (2011) afirman que, tanto en el mercado como en el Estado, el aumento de las redes de distribución ha coincidido con un giro marcado hacia lo humano, lo personal y lo individual.

Así entonces, la transferencia del conocimiento se define como el proceso por medio del cual se traspasa el conocimiento, no sólo dentro, sino también fuera de las sociedades. De ahí que, las universidades puedan intervenir activamente en tres aspectos dentro de este proceso de interacción con las empresas, según Bayona y González (2010):

1. Generando nuevo conocimiento científico básico y también aplicado a través de sus propios grupos de investigación con el apoyo del sector público y privado.

2. Dando formación con alta calidad de científicos, ingenieros, técnicos, administradores y demás profesionales, que satisfagan las demandas del comercio y la industria. 
3. Cristalizando los avances de la investigación, creando una red de empresas industriales y de nuevos negocios.

A partir de esos ejes de trabajo, en el LIS entendemos y relacionamos la Innovación Social, como un eje investigativo, un campo de acción transversal y dinamizador, en el marco de la cuarta revolución industrial.

Dentro de estas líneas de trabajo, se establece el modelo de flujo de datos del laboratorio LIS, el cual nace como resultado de los esfuerzos mancomunados para la creación de una plataforma de analítica interdisciplinar, desde el pienso ingenieril y con impacto social que puede servir a organizaciones e instituciones, en donde los datos al ser transformados en información pueden contar historias, convirtiéndose en una fuente de toma de decisiones confiable, como se muestra en la Figura 2.

Figura 2. Modelo de flujo de datos del laboratorio LIS

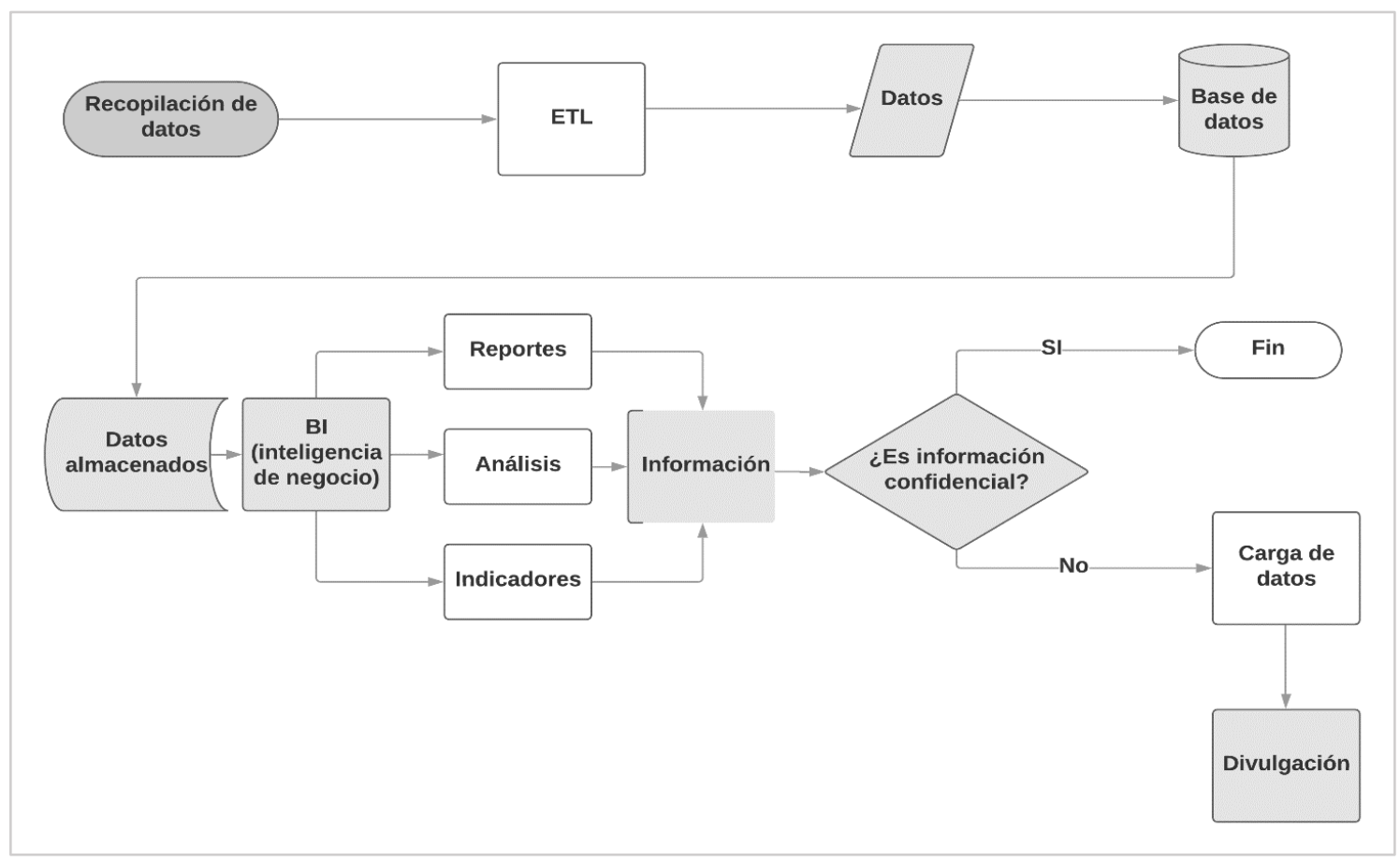

Fuente: Elaboración propia, 2020.

En la Figura 2, se ha relacionado el enfoque de análisis de grandes conjuntos de datos (Big Data) suscrito a uno de los propósitos de la innovación social (espacios de co-creación) con la finalidad de generar diferentes casos de uso de los datos, posibilitando el descubrimiento de información, en volumen y correlación proveniente de fuentes estructuradas y no estructuradas.

5. Innovación, creatividad o adaptación de herramientas administrativas o tecnológicas que desarrollen o mejoren el proceso

Como espacio de innovación, en el LIS, se ha liderado el desarrollo de proyectos académicos e investigativos, de aplicabilidad, proyección e impacto social. Así mismo se ha participado en el diseño e implementación de programas, proyectos y acciones, al igual que en la ejecución de contratos y convenios, celebrados con dependencias públicas del ente territorial, departamental y nacional, en coordinación con la Vicerrectoría de Docencia e Investigación y en particular, con la dirección Operativa de Extensión del ITM. 
De acuerdo con la Misión y Visión del Laboratorio, y los niveles de desarrollo que ha tenido dentro de la institución y el municipio, se han acompañado y desarrollado importantes proyectos, en articulación con los Planes de Desarrollo municipal, regional, Nacional y que se reasumen dentro de los Planes de Desarrollo institucional, ITM desde 2012 y proyectados a 2019, como acciones innovadoras y de adaptación y aplicabilidad en distintos contextos de la ciudad, la región y el país entre los cuales podemos destacar los que se presentan a continuación.

\subsection{Proyecto Música para la Paz-LATIS (Municipios del Tolima)}

Se basó en el desarrollo de talleres realizados para co-crear comunitariamente, mediante el uso de la metodología de Diseño Centrado en las Personas, cuatro Laboratorios de Arte y Tecnología para la Innovación Social -LATIS, en diversos municipios de Colombia. Dicha cocreación parte de recrear los diversos imaginarios urbanos, sociales y culturales, que las personas y las comunidades utilizan para explicar sus condiciones de vida e implica identificar los componentes pedagógicos, artísticos, socioculturales, materiales y funcionales, que darán forma y vida a los LATIS.

\subsection{Proyecto: Arte para el desarrollo (Ituango - Antioquia)}

Este proyecto se desarrolló en el municipio de Ituango Antioquia, en el área de la educación, arte y cultura, enmarcado en la implementación de nuevos mecanismos de aprendizaje con elementos experimentales -participativos de innovación social, para el fortalecimiento del modelo pedagógico utilizado y que viene siendo liderado en el municipio por la Institución educativa Pedro Nel Ospina.

Tuvo como objetivo promover la educación desde el arte y la cultura como agentes generadores del desarrollo endógeno del municipio de Ituango en el Departamento de Antioquia, formando y educando las emociones para consolidar una comunidad más tolerante, deseosa y amante de la paz; conocedora, crítica y capaz de generar un clima de confianza para hacer frente a los problemas y tensiones que traería aparejado un posible y deseable postacuerdo colombiano.

5.3. Proyecto: Formación ciudadana y construcción de sujetos políticos en Instituciones de Educación Superior adscritas al municipio de Medellín, para superar las prácticas de la cultura de la ilegalidad en el ámbito de educación superior

La convocatoria "Concurso de Méritos Abierto - № CMA-001-2014" realizada por Sapiencia, tuvo como finalidad, generar conocimiento en legalidad en la educación superior, enmarcado en el ámbito de la Cultura Política y la Ética Educativa, teniendo en cuenta patrones de orientación, actitudes, normas y creencias compartidas en el municipio de Medellín.

En este proyecto se realizó un análisis de las normas, leyes, decretos y/o resoluciones que hacen posible la administración de las tres IES adscritas al municipio de Medellín. Así mismo, se tipificaron las experiencias de ilegalidad que se practican en el desarrollo y cumplimientos de dichas normas.

Se trabajó con toda la comunidad académica de las tres IES: estudiantes, docentes y personal administrativo, directivos. Se aplicaron encuestas de percepción y definición de las situaciones de riesgo y prácticas de ilegalidad. Así mismo se realizaron entrevistas semiestructuradas a directivos y docentes, como muestras aleatorias y grupo de expertos. En total fueron: 350 estudiantes y 70 docentes encuestados y 12 directivos entrevistados. 
5.4. Proyecto: Programa de transición y mejora de la articulación entre la Educación Media y la Educación Superior - población estudiante

Como uno de los factores de la baja calidad académica de la Educación Superior, se aduce, que hay un bajo nivel de formación en la Básica Secundaria. Pero en especial, es evidente que en Colombia no podemos hablar de un Sistema Educativo. Hay una desarticulación, falta cohesión en los niveles de formación, para lograr los niveles de desarrollo y calidad educativa, de acuerdo a los estándares nacionales, latinoamericanos y en particular de la OCDE.

Bajo este panorama, el programa de gobierno 2016-2019 de la administración del municipio de Medellín se propuso el acompañamiento y articulación de la educación superior con los niveles de formación de básica secundaria, en el marco de la formación técnica y jornada complementaria y única. Así mismo, y en relación con lo planteado en el Plan de Desarrollo Municipal, el Plan de Desarrollo 2016-2019 del ITM en el eje tres “Extensión y proyección social para fortalecer vínculos Institución - Sociedad" a tráves de dos programas: 1. Programa de cualificación para la excelencia docente en los niveles precedentes, 2. Programa de transición y mejora de la articulación entre la educación media y la educación. Desde el LIS se lideran los procesos de cualificación para los docentes de las instituciones educativas, el acompañamiento a los estudiantes en su preparación para las distintas pruebas, así como el fortalecimiento de competencias en Ciencias Básicas y Habilidades Comunicativas. Para dar cumplimiento a este componente del Plan de Desarrollo, se diseñaron una serie de estrategias que van desde el diseño e implementación de seminarios, talleres, diplomados hasta el componente central para la toma de decisiones que es la caracterización a modo de diagnóstico institucional a partir de una muestra de estudiantes de los grados $9^{\circ}$ y $10^{\circ}$.

\section{Conclusiones}

Hablar de innovación social remite a comprender la manera en que acciones con cierto grado de novedad toman fuerza en torno a la satisfacción de las necesidades sociales, lo cual implicará pensar en la participación activa de actores de diverso orden que a partir de su experiencia y saber aporten a la construcción significativa de soluciones a las diferentes problemáticas detectadas. Así, el trabajo del LIS desde los ámbitos de las prácticas sociales, apropiación social, innovación social y transferencia social, erige una proyección en el trabajo potencial del desarrollo tecno-científico desde las cosas simples que constituyen la cotidianidad de las comunidades a través de acciones de co-creación y eco-creación.

En consecuencia, lo que corresponde al LIS en materia de lo qué hace y está en capacidad de hacer, cómo lo hace y las líneas de trabajo del laboratorio, es evidente afirmar que todas sus acciones son replicables en distintas regiones. Consideramos en este sentido, que es necesario tener presente las particularidades de cada sector, comunidad, región o territorio, lo cual será la base del éxito de toda práctica social.

Como unidad investigativa sustentada en grupos de investigación y programas formativos, es claro que dispone de manera permanente de un recurso humano, cualificado y capacitado para llevar a cabo las acciones declaradas en su misión y los planes estratégicos que como unidad administrativa se propone.

Finalmente, desde la aplicabilidad a los programas y proyectos internos y externos, vemos como las distintas metodologías implementadas en el desarrollo de las diversas acciones, dejan en evidencia y hacen posible que las mismas sean replicadas de manera permanente en todos los planes, programas, proyectos y estrategias que se llevan a cabo en el ITM, la ciudad, la región y el país. En igual medida, vemos que las metodologías de corte colaborativo, cooperativo, de co-creación y eco-creación son herramientas metodológicas para generar capacidades y potenciar procesos de desarrollo en los grupos sociales. 


\section{Referencias}

Banco Interamericano de Desarrollo - BID. (2017). Innovation Lab (I-Lab). Washington D.C. Disponible en: https://www.bidinnovacion.org/es/

Bayona, C. \& González, R. (2010). La transferencia de conocimiento en la Universidad Pública de Navarra. Pamplona: Universidad Pública de Navarra.

Colegio Mayor de Antioquia. (2018). Desarrollo técnico de la propuesta Laboratorio de Innovación social. Medellín: Colegio Mayor de Antioquia. Disponible en: https://www.colmayor.edu.co/laboratorio-deinnovacion-social/

De la Mata, G. (2011). Manual de Innovación Social: De la idea al proyecto. Disponible en: http://www.economicas.unsa.edu.ar/afinan/informacion general/book/ebooks/manual-de-innovacionsocial-guadalupe-de-la-mata.pdf

Fajnzylber, F. (1990). Industrialización en América Latina: de la caja negra al casillero vacío: comparación de patrones contemporáneos de industrialización. Santiago de Chile: Comisión Económica para América Latina y el Caribe - CEPAL.

Giraldo Gutiérrez, F. L., Ortiz-clavijo, L. F. \& Zuñiga-Miranda, S. (2020). Políticas de Ciencia, Tecnología e Innovación en América Latina y el Caribe y su influencia en la producción y apropiación de la CTI. Revista Linguagem \& Ensino, 23(1), 296-316. DOI: https://doi.org/10.15210/RLE.V23I1.17751

Instituto Tecnológico Metropolitano - ITM. (2019). Laboratorio de Innovación Social - ITM. Disponible en: https://www.itm.edu.co/facultades/facultad-de-artes-y-humanidades-18/centro-de-investigacion-yextension/laboratorio-de-innovacion-social/

Jovesólides. (2020). Encouraging social entrepreneurship among european youth. Disponible en: https://europa.eu/youth/volunteering/organisation/947059501 it

Monge, N. \& Allamand, A. (2016). Innovación Social y valor compartido: el cambio de paradigma de la intervención social empresarial. En: D. Domanski, N. Monge \& D. Rocha (eds.), Innovación Social en Latinoamérica (pp. 71-92). Bogotá: Corporación Universitaria Minuto de Dios.

Muñoz, R. (2017). Seis canastas para innovar. El método revolucionario que pondrá a la innovación al alcance de todos. Barcelona: Grijalbo.

Murray, R., Mulgan, G. \& Caulier-Grice, J. (2011). How to Innovate: The tools for social innovation. Londres: The Young Foundation.

Serra, A. (2010). Citilabs: ¿Qué pueden ser los laboratorios ciudadanos? La Factoría. Disponible en: https://revistalafactoria.org/articulos/citilabs-laboratorios-ciudadanos

Universidad Nacional de Colombia. (2018). Programa de Innovación social. Bogotá: Universidad Nacional de Colombia.

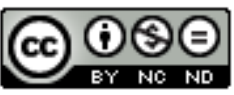

(C) Attribution-NonCommercial-NoDerivatives 4.0 International (CC BY-NC-ND 4.0)

https://creativecommons.org/licenses/by-nc-nd/4.0/ 